\title{
Highly Efficient Nitration of Phenolic Compounds in Solid Phase or Solution Using $\mathrm{Bi}\left(\mathrm{NO}_{3}\right)_{3} \cdot \mathbf{5 H}_{2} \mathrm{O}$ as Nitrating Reagent
}

\section{Hong-Bin Sun, ${ }^{a, b}$ Ruimao Hua, ${ }^{* a}$ Yingwu Yin ${ }^{* b}$}

${ }^{a}$ Department of Chemistry, Tsinghua University, Key Laboratory of Organic Optoelectronics \& Molecular Engineering of Ministry of Education, Beijing 100084, China.

${ }^{b}$ Key Laboratory of Bioorganic Phosphorus Chemistry of Ministry of Education, Beijing 100084, China.

ruimao@mail.tsinghua.edu.cn (Ruimao Hua)

\section{Supporting Information:}

1. General Method and MS data (p S2-S4)

2. ${ }^{1}$ H-NMR charts of the nitrated products(p S5-S19) 


\section{General Methods}

All the products are known compounds, which were identified by GCMS and ${ }^{1} \mathrm{H}-\mathrm{NMR}$ spectra (in $\mathrm{CDCl}_{3}$, at $300 \mathrm{MHz}$ and the chemical shifts $(\delta)$ were referenced to solvent resonances or TMS).

GC-MS $m / z$ (\% rel. inten.)

2-Nitrophenol (Table 1, entry 1): 139 ( $\left.\mathrm{M}^{+}, 40\right), 109$ (35), 81 (55), 65 (60), 53 (38), 39 (100), $30(66)$.

4-Nitrophenol (Table 1, entry 1): 139 ( $\left.\mathrm{M}^{+}, 15\right), 109$ (55), 81 (30), 65 (86), 53 (33), 46 (22), 39 (100), 30 (76), 28 (41).

4-Methyl-2-nitrophenol (Table 2, entry 1): 153 ( $\left.\mathrm{M}^{+}, 91\right), 137$ (4), 123 (25), 107 (17), 95 (29), 77 (100), 65 (24), 51 (45), 39 (25), 30 (17).

4-Nitro-2-methylphenol (Table 2, entry 3): 153 ( $\left.\mathrm{M}^{+}, 38\right), 137$ (12), 123 (51), 108 (5), 77 (100), 53 (34), 39 (26), 30 (20), 28 (75).

6-Methyl-2-nitrophenol (Table 2, entry 3): $153\left(\mathrm{M}^{+}, 10\right), 137$ (2), 107 (6), 77 (20), 53 (14), 39 (9), 28 (100).

6-Methyl-2, 4-dinitrophenol (Table 2, entry 4): 198 ( $\left.\mathrm{M}^{+}, 100\right), 168$ (44), 152 (9), 121 (68), 105 (53), 93 (35), 77 (44). 
5-Methyl-2-nitrophenol (Table 2, entry 5): 153 ( $\left.\mathrm{M}^{+}, 88\right), 137$ (18), 123 (77), 108 (11), 105 (11), 95 (42), 77 (100), 65 (32), 55 (14), 51 (43), 39 (34), 28 (20).

3-Methyl-4-nitrophenol (Table 2, entry 5): 153 ( $\left.\mathrm{M}^{+}, 4\right), 136$ (5), 123 (9), 81 (3), 77 (8), $51(8), 39(6), 28$ (100).

4-Nitro-1, 3-benzenediol (Table 2, entry 6): $155\left(\mathrm{M}^{+}, 6\right), 139$ (32), 121 (7), 109 (79), 77 (19), 65 (47), 51 (19), 39 (48), 30 (25), 28 (100).

2-Chloro-4-nitrophenol (Table 2, entry 7): $173\left(\mathrm{M}^{+}, 11\right), 143$ (22), 107 (8), 99 (12), 79 (16), 63 (23), 51 (10), 28 (100).

2-Chloro-6-nitrophenol (Table 2, entry 7): $173\left(\mathrm{M}^{+}, 49\right), 156$ (4), 143 (59), 126 (7), 115 (41), 99 (36), 73 (24), 63 (100), 51 (16), 37 (11), 30 (25).

4-Chloro-2-nitrophenol (Table 2, entry 8): 173 ( $\left.\mathrm{M}^{+}, 64\right), 156$ (7), 143 (26), 128 (10), 115 (41), 99 (39), 73 (29), 63 (100), 51 (20), 37 (13), 30 (26).

4-Hydroxy-3-nitro-benzaldehyde (Table 2, entry 10): $167\left(\mathrm{M}^{+}, 71\right), 166$ (100), 120 (27), 109 (3), 92 (20), 81 (11), 63 (47), 53 (24), 39 (28), 30 (14). 
2, 4-Dinitrophenol (Table 2, entry 11): $184\left(\mathrm{M}^{+}, 61\right), 154$ (100), 107 (47), 91 (35), 79 (44), 63 (66), 51 (24), 30 (41).

Methyl 4-hydroxy-3-nitrobenzoate (Table 2, entry 12): $197\left(\mathrm{M}^{+}, 29\right), 167$ (12), 166 (100), 136(9), 120 (34), 119 (12), 108 (7), 92 (14), 91 (12), 77 (6), 63 (33), 51 (7), 30 (15), $28(15)$. 
2. ${ }^{1} \mathrm{H}-\mathrm{NMR}$ charts of the nitrated products 


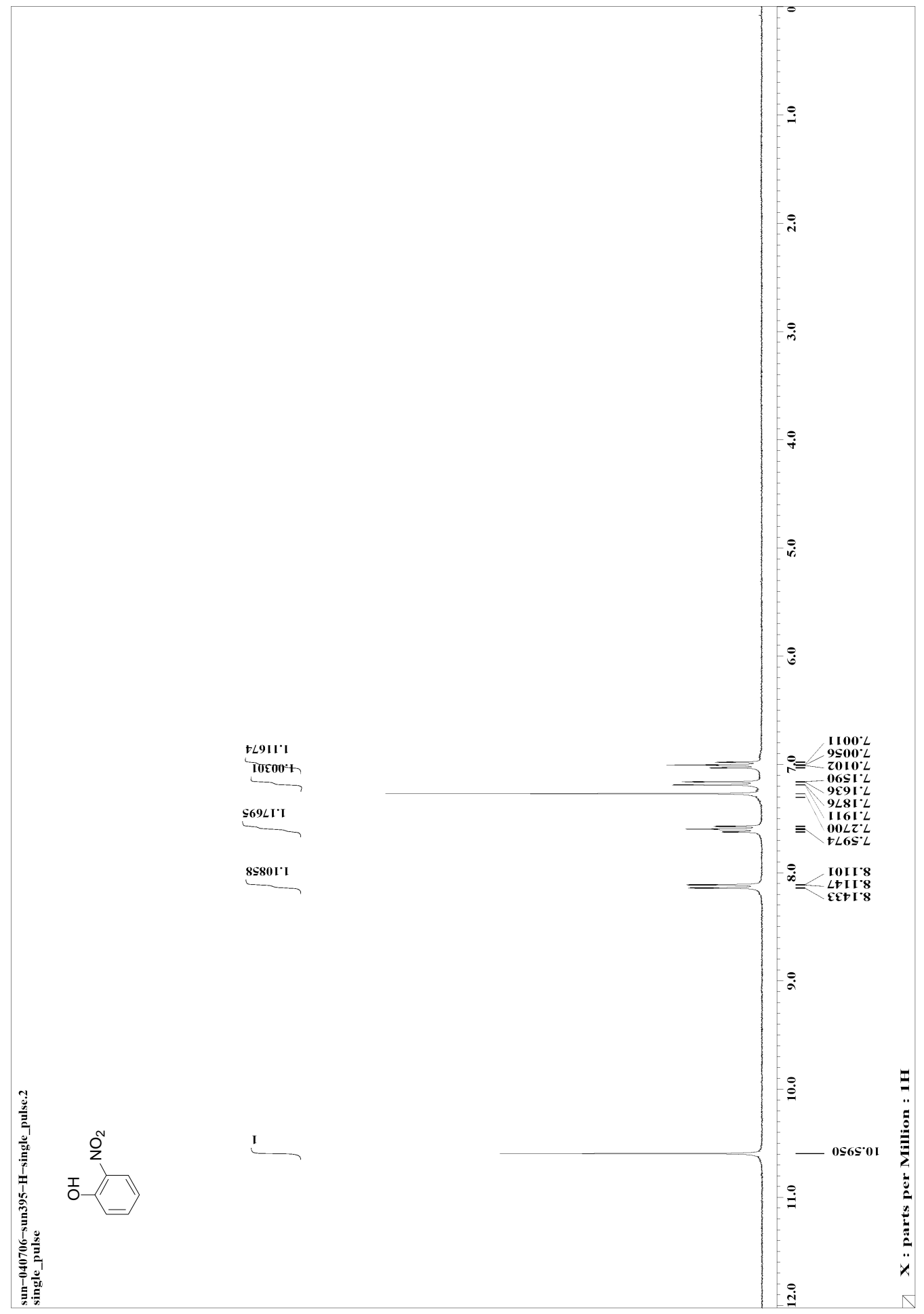




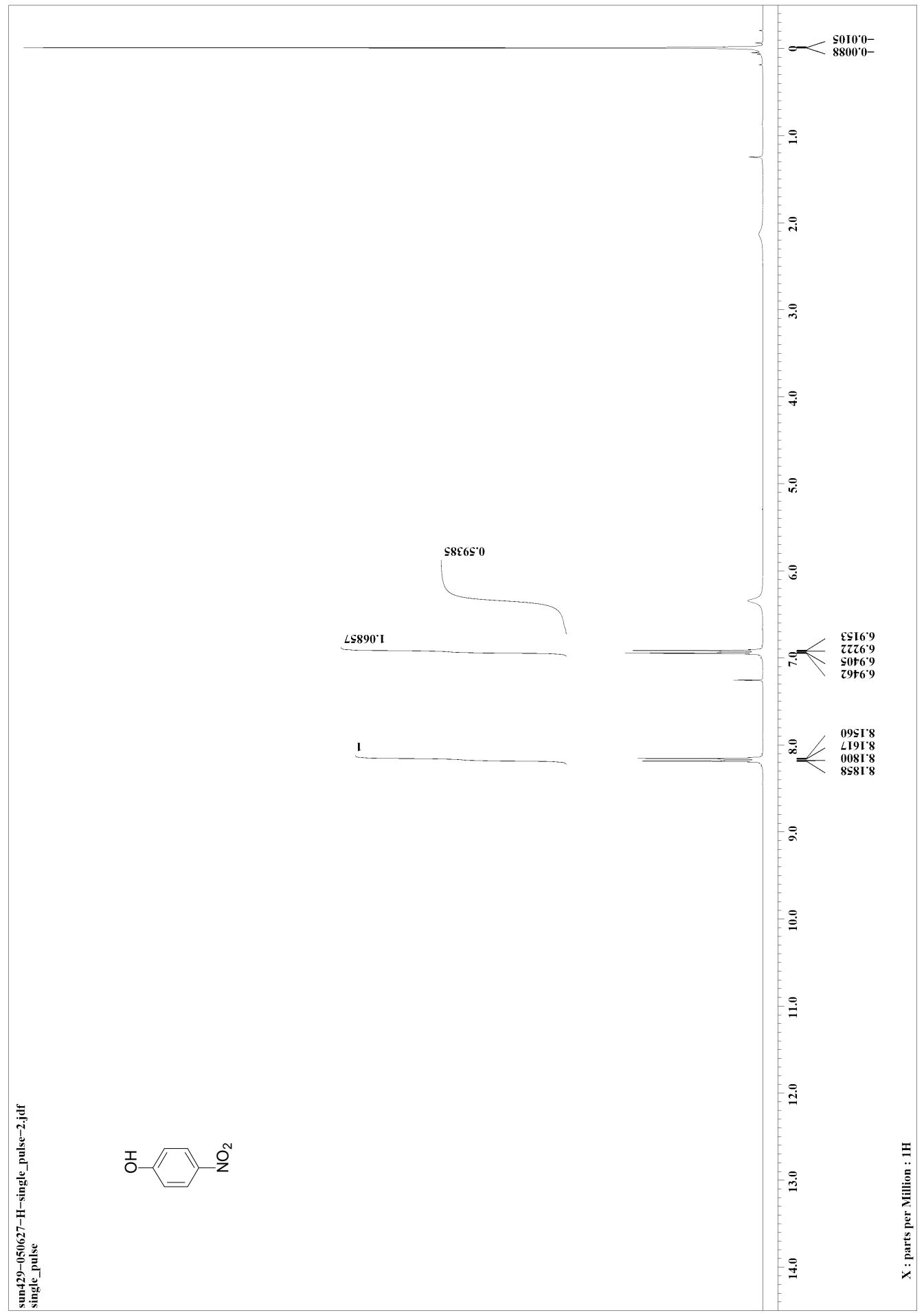




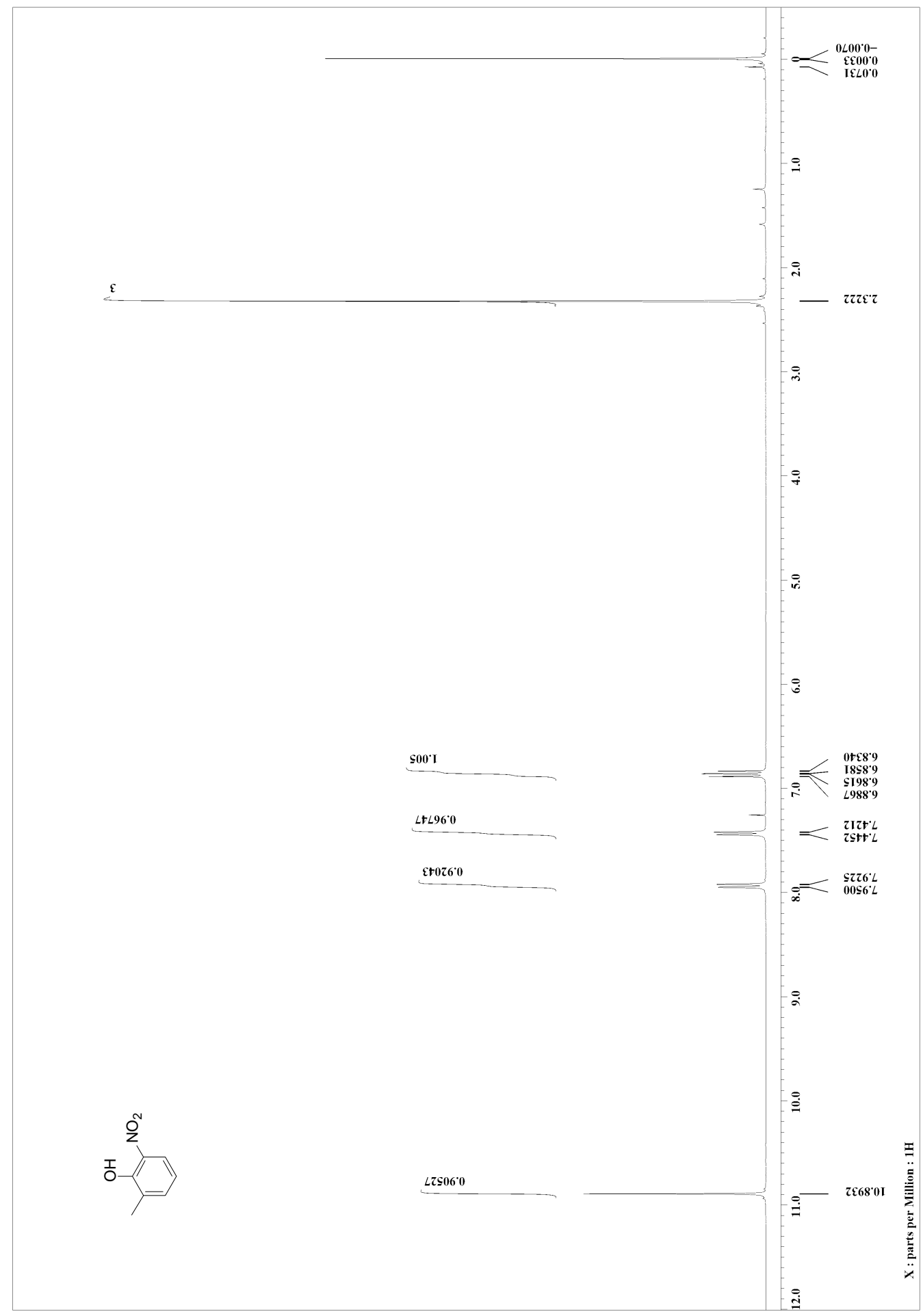




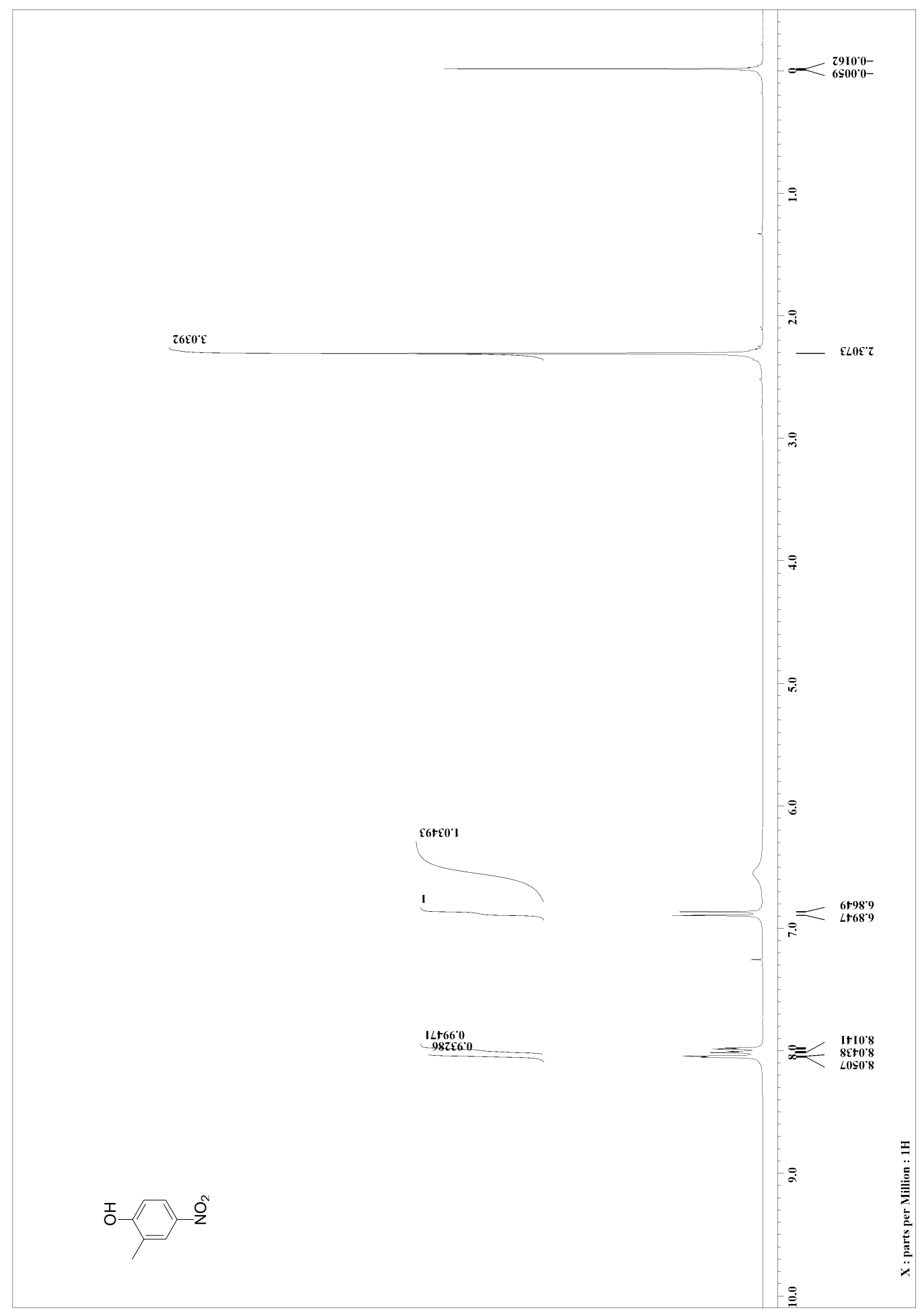




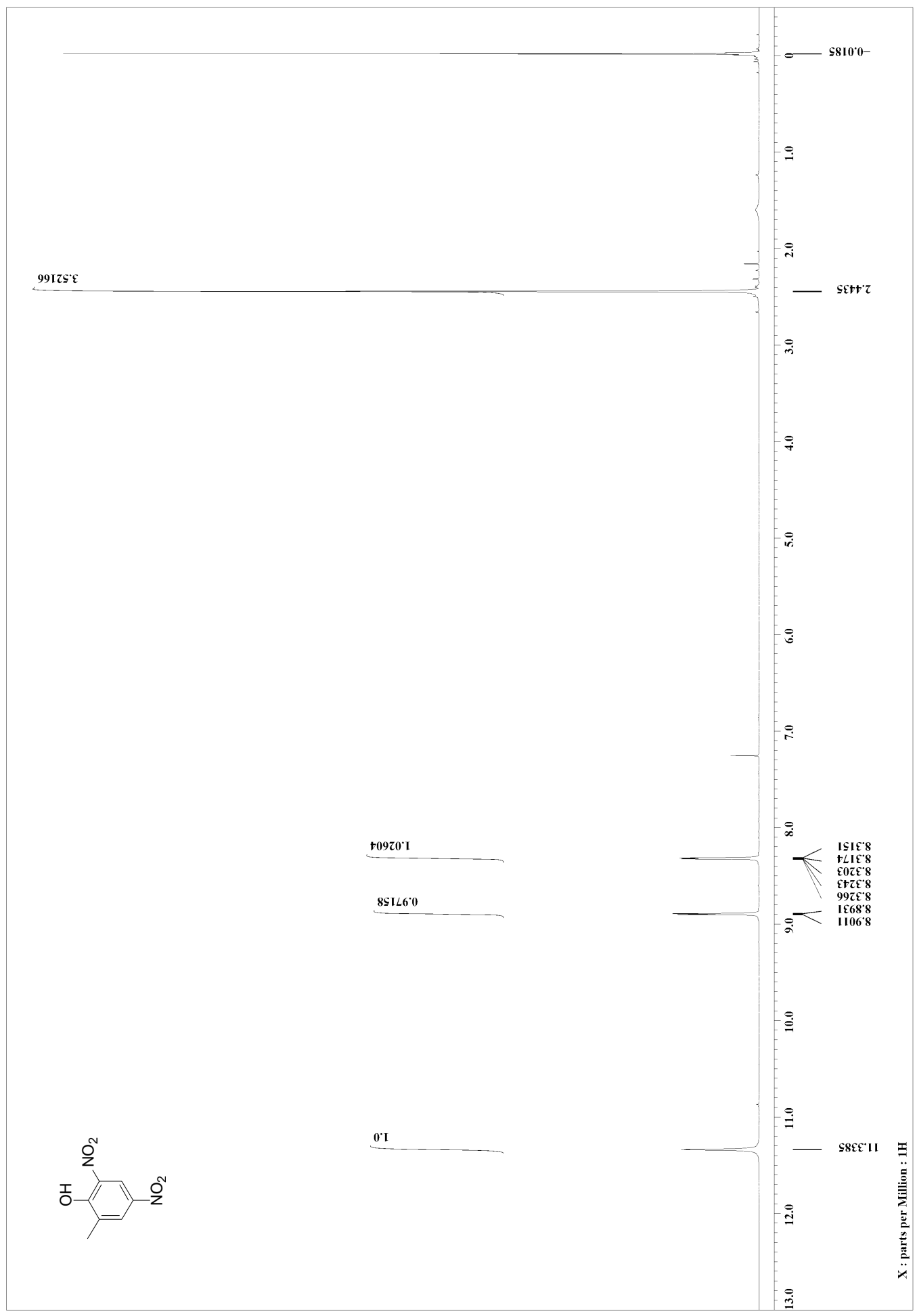

$\stackrel{2}{n}$ 


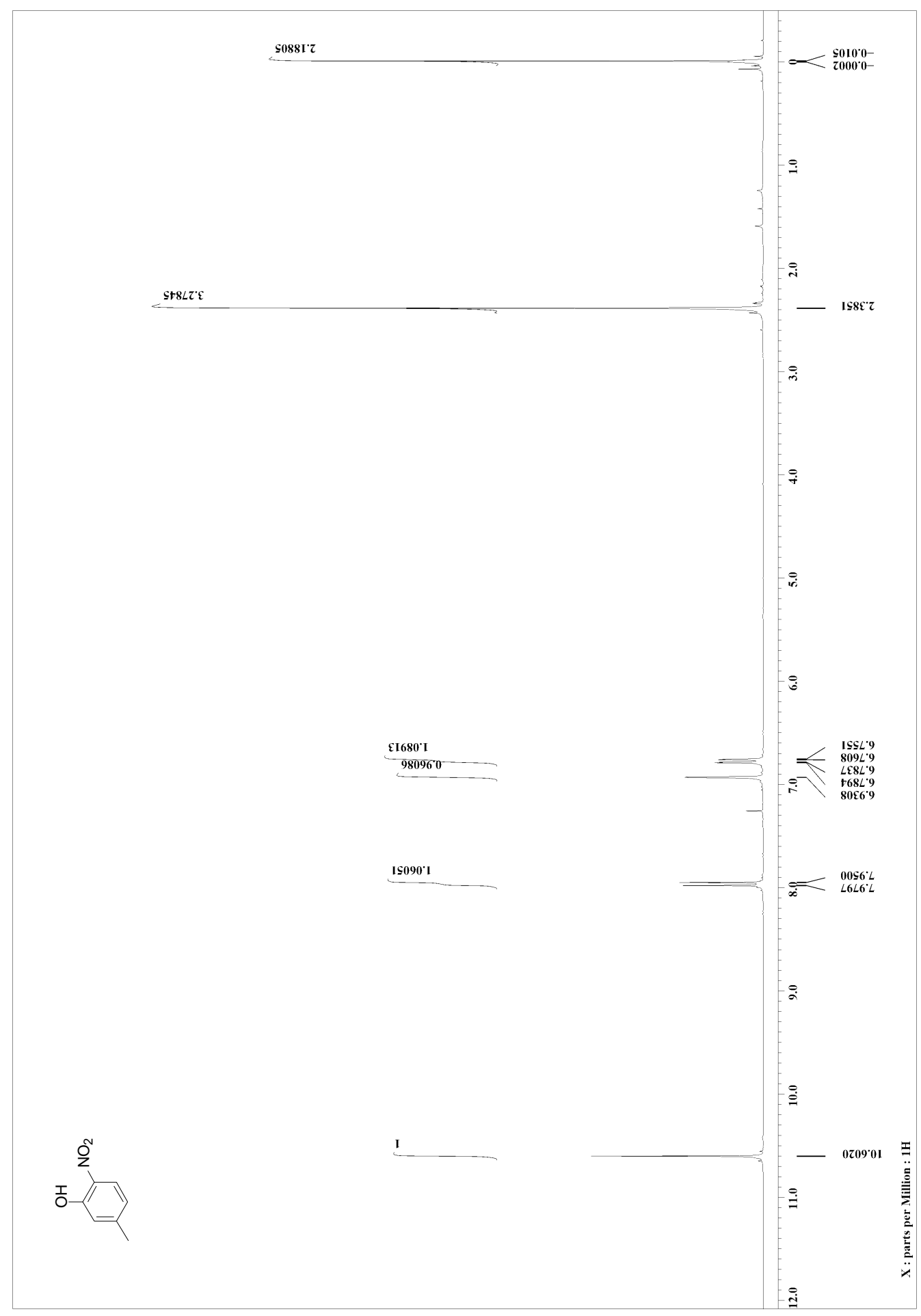

$\bar{n}$ 


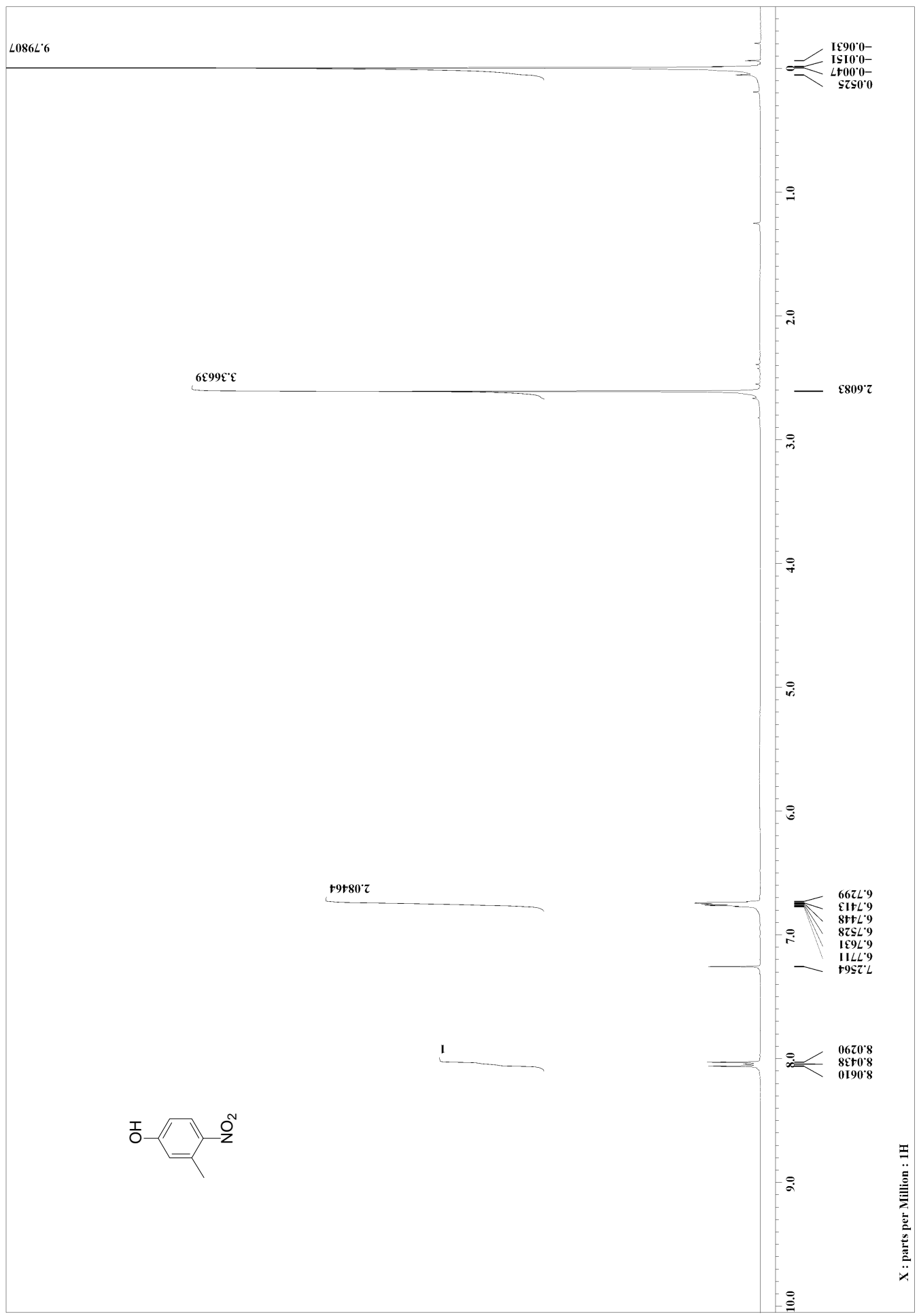




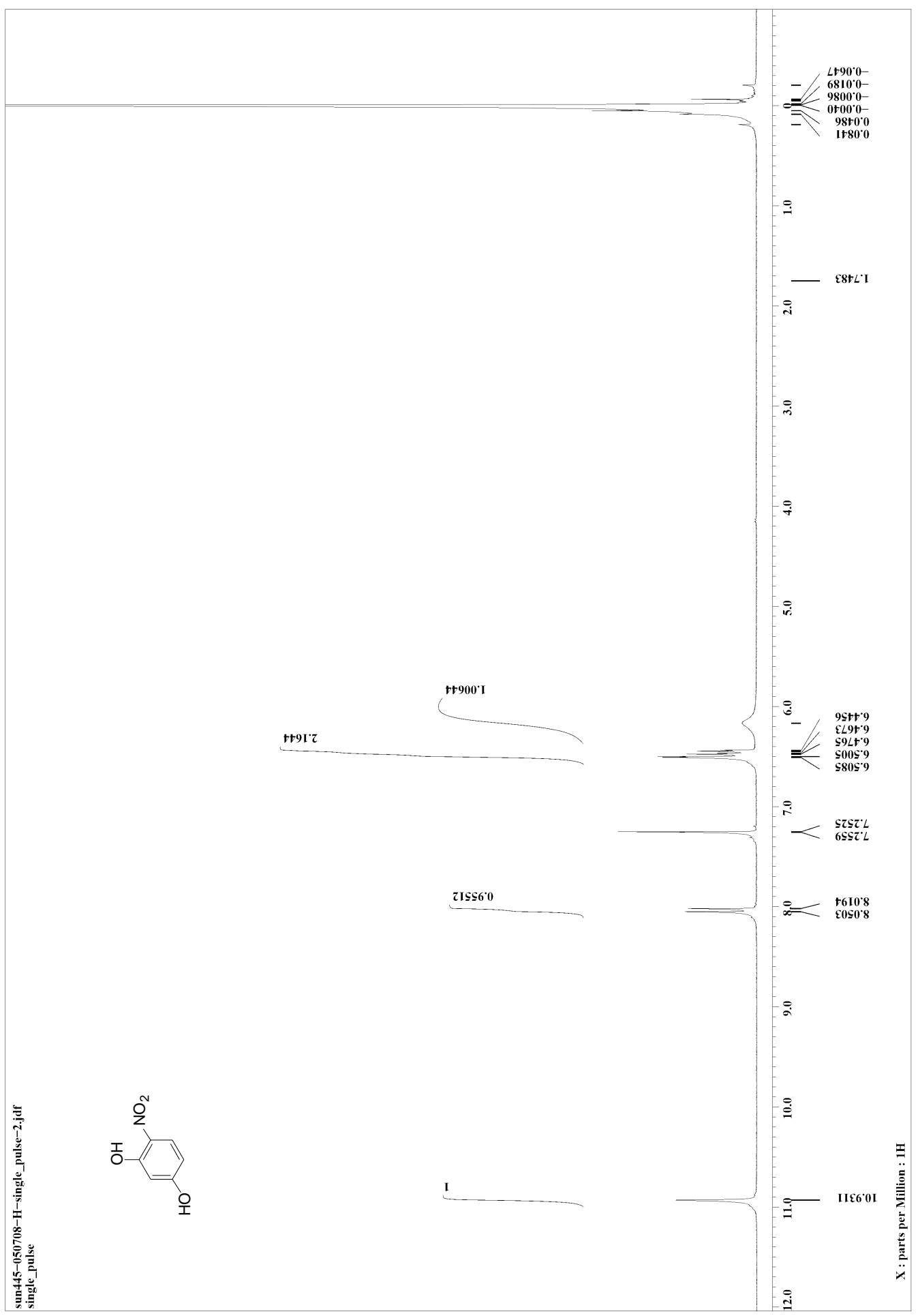




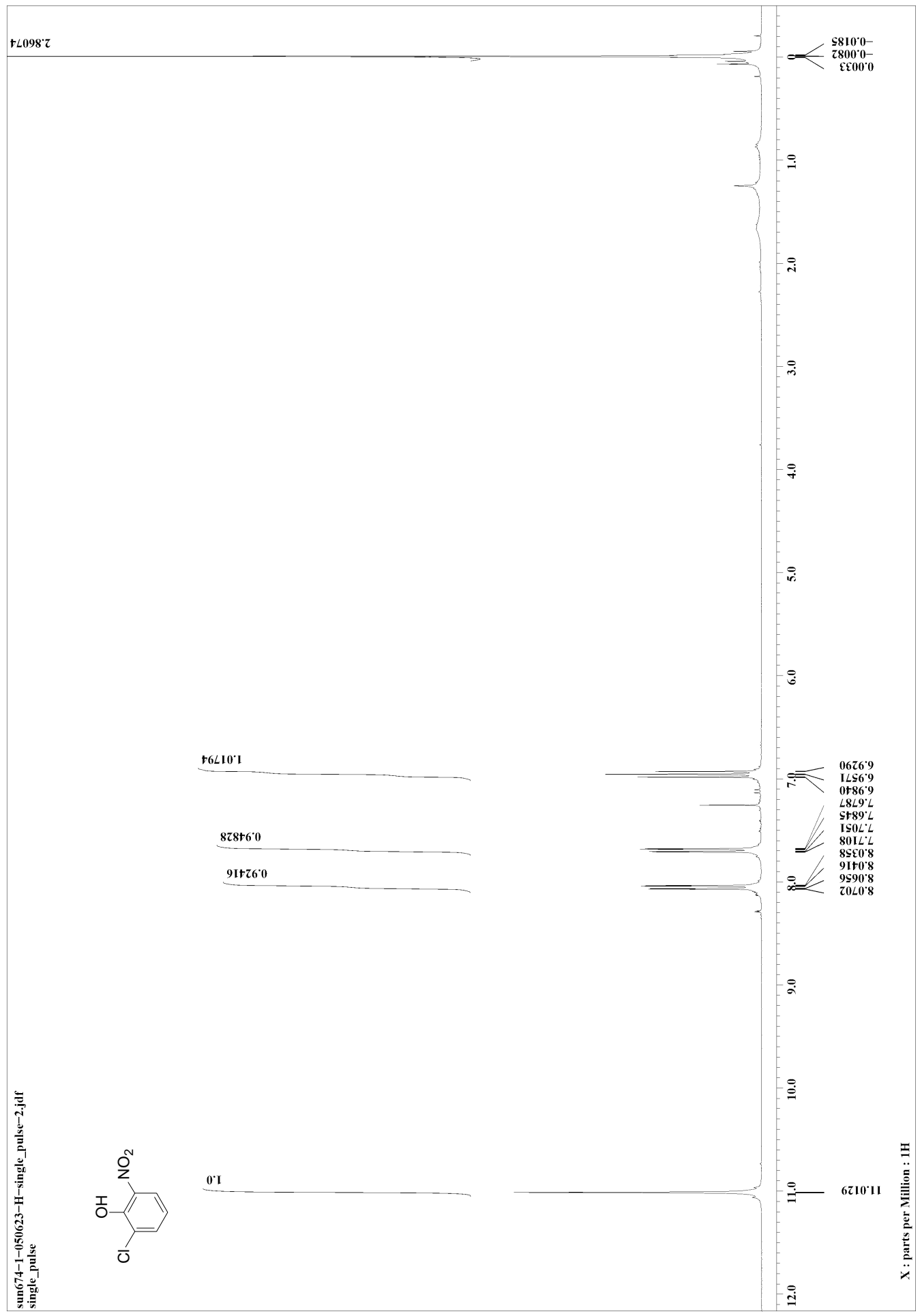

$\frac{t}{n}$ 


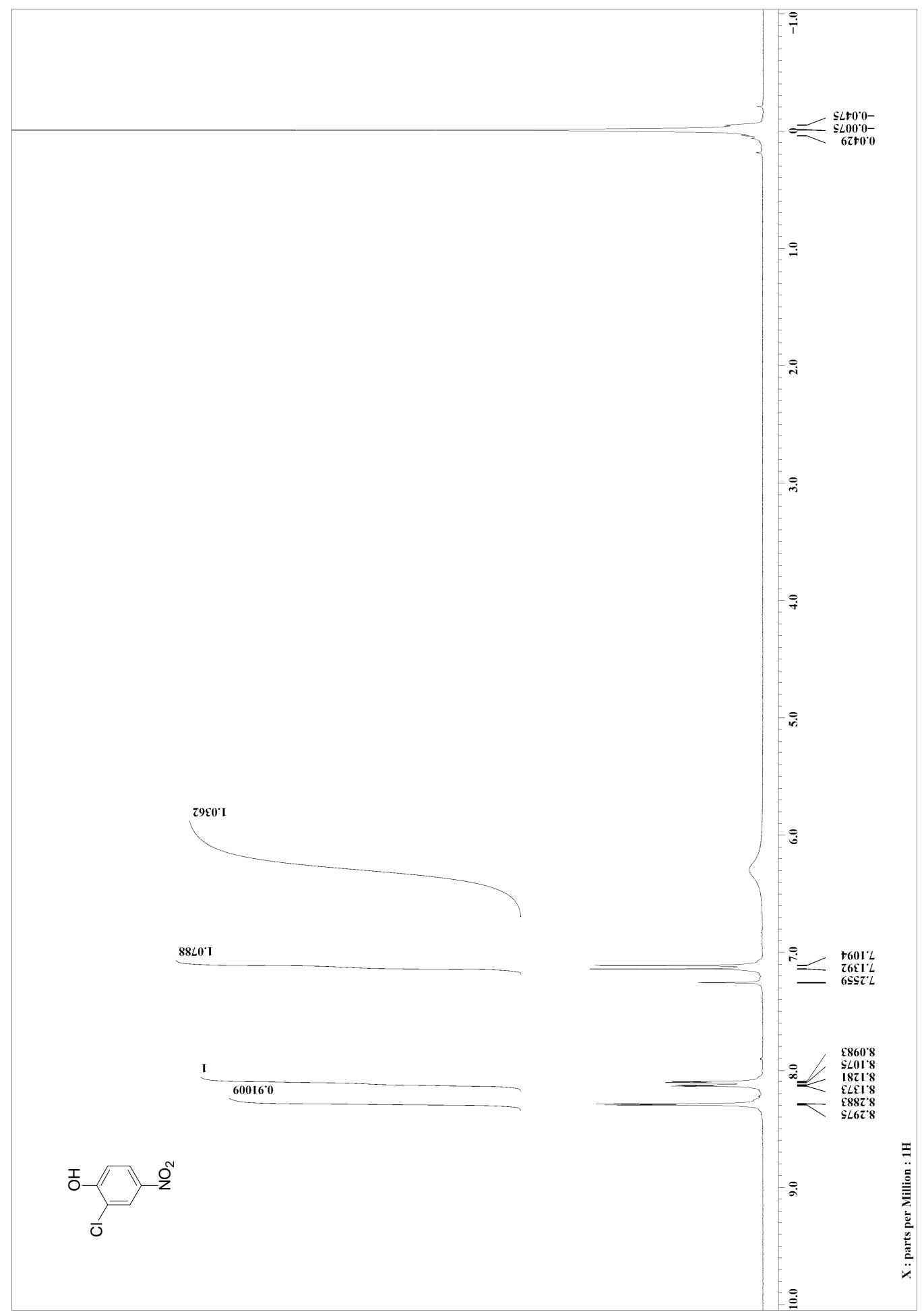

$\frac{n}{n}$ 


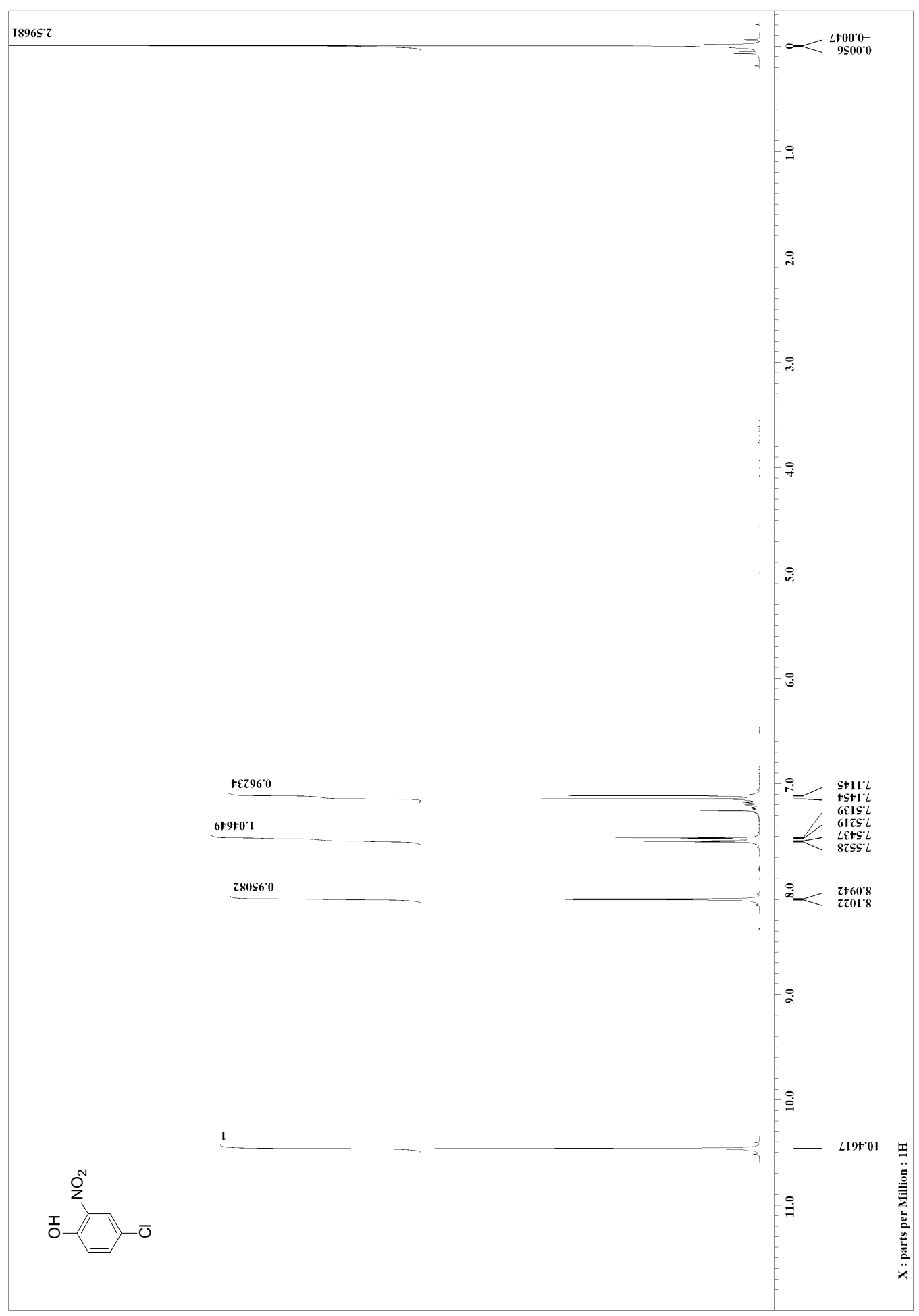




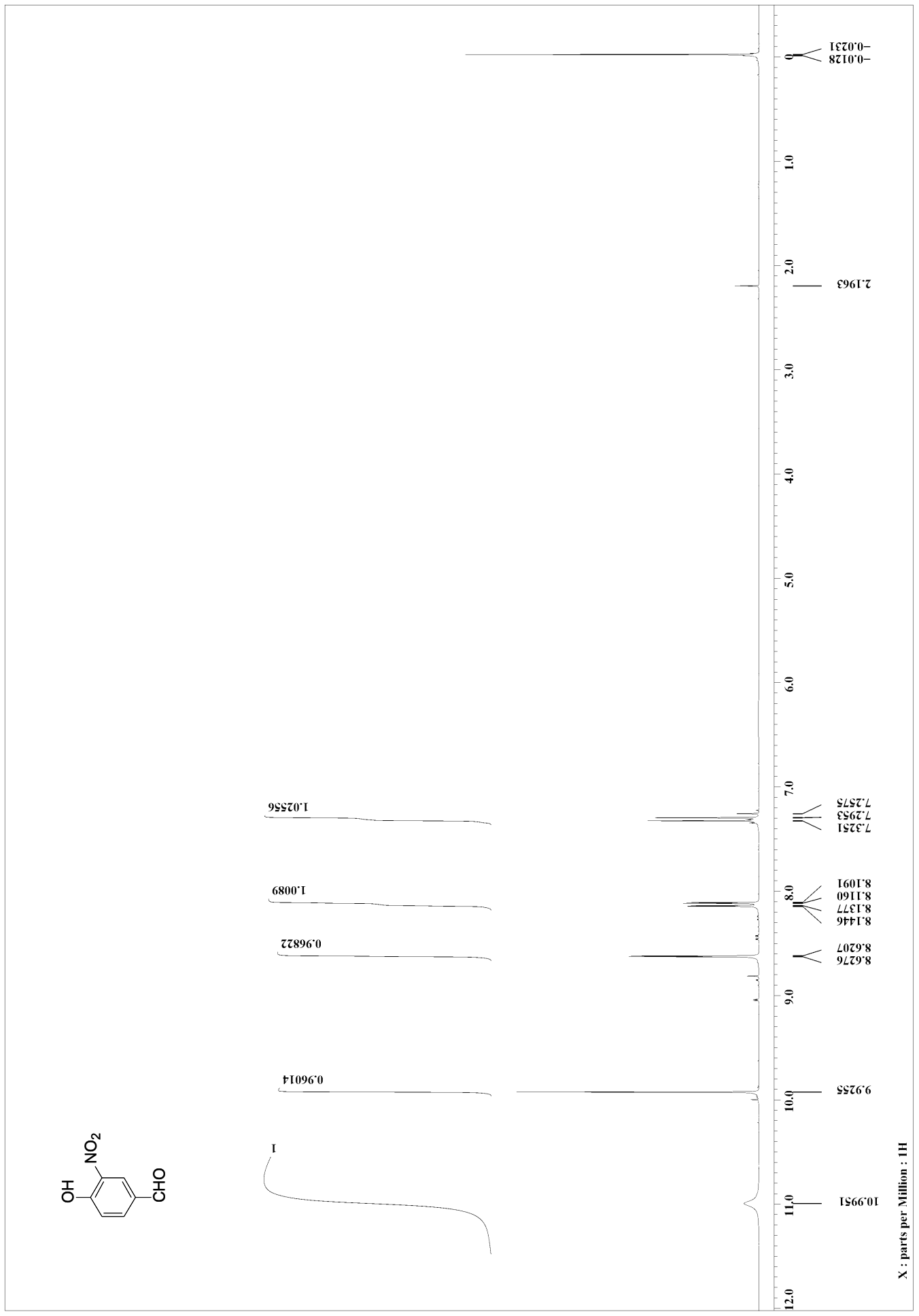

$\frac{1}{n}$ 


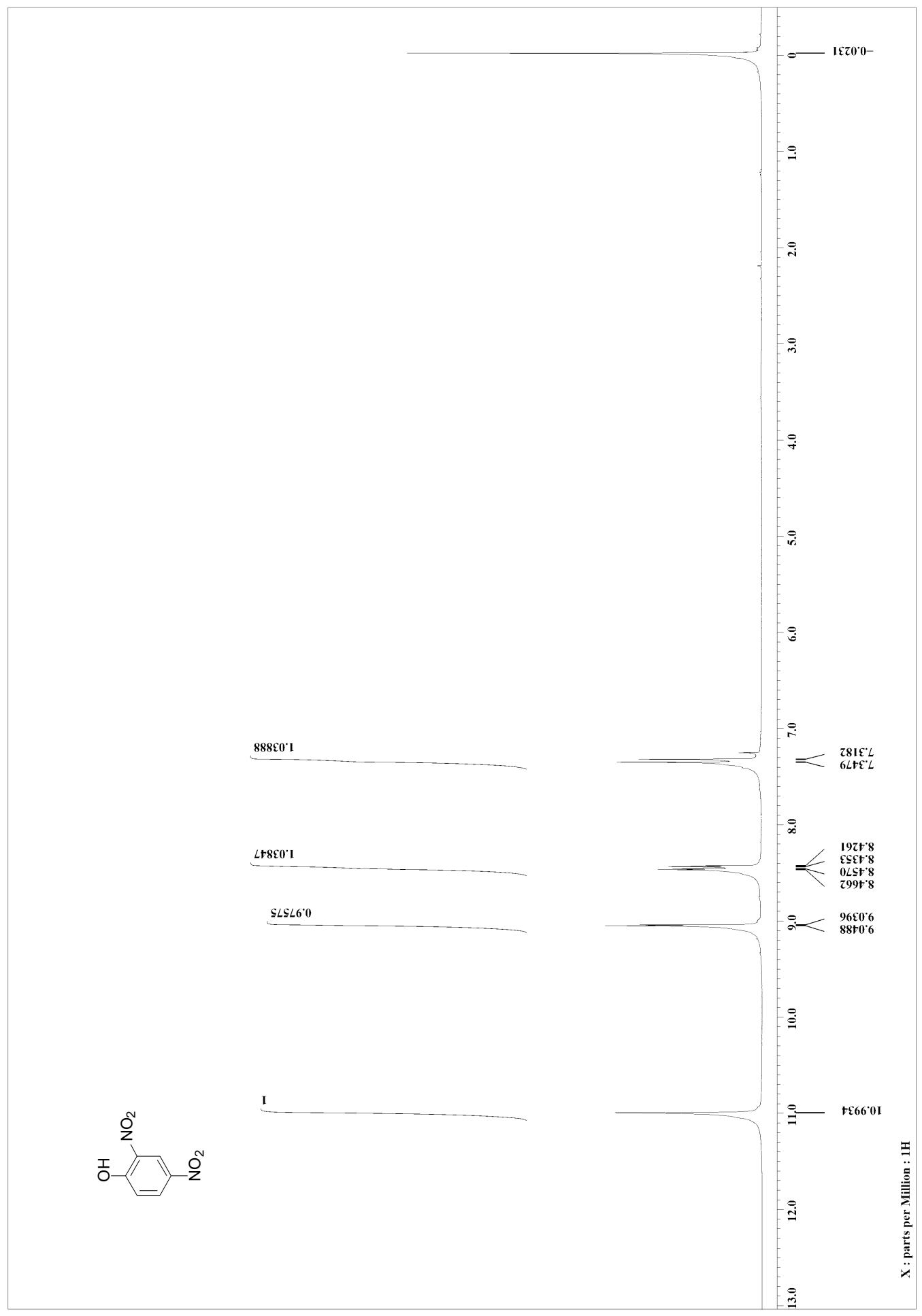




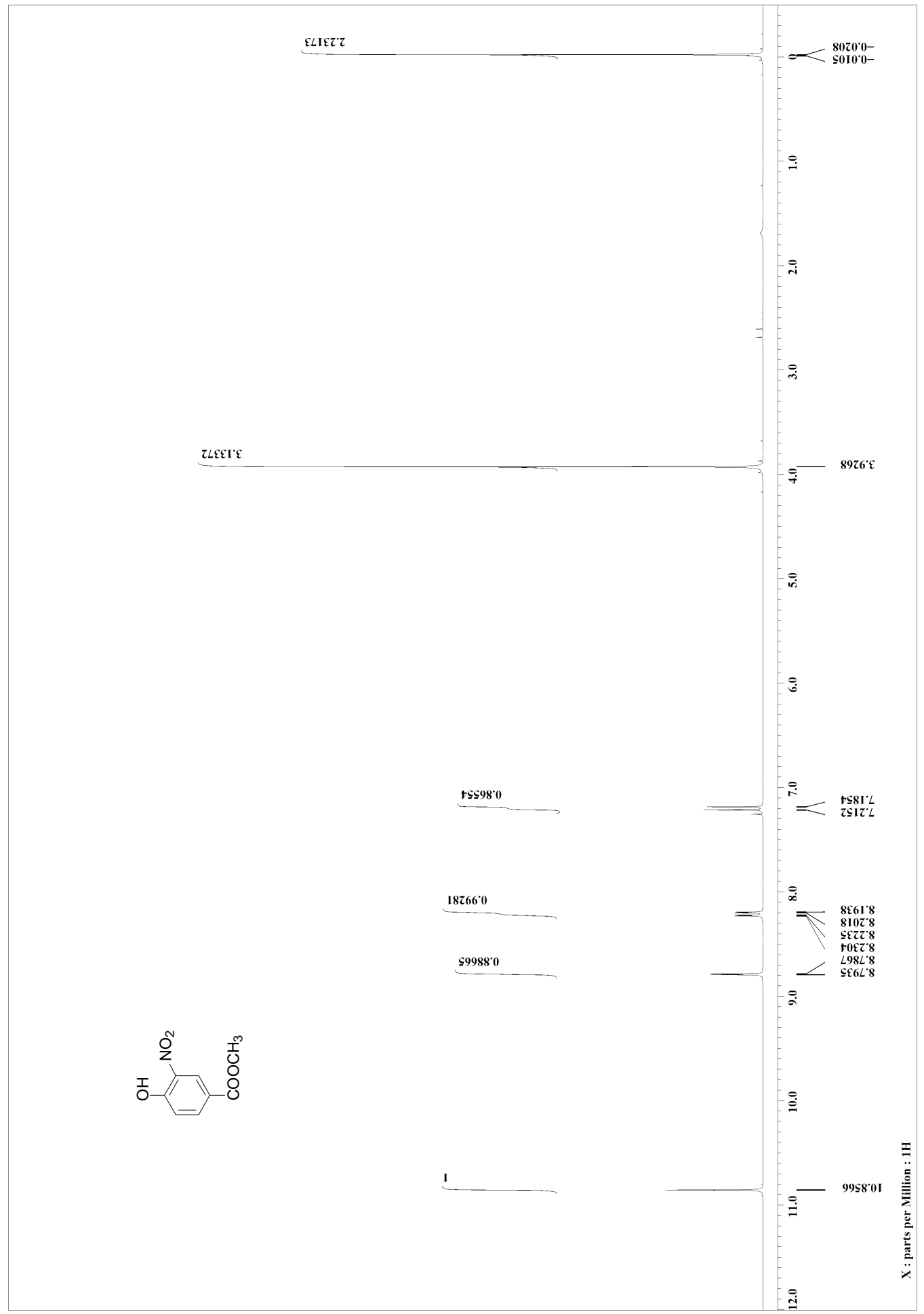

के 